\title{
Reproducibility of aortic intima-media thickness in infants using edge-detection software and manual caliper measurements
}

Kate McCloskey ${ }^{1,2,3}$, Anne-Louise Ponsonby ${ }^{1,3+}$, John B Carlin ${ }^{1}$, Kim Jachno ${ }^{1}$, Michael Cheung ${ }^{1,3}$, Michael R Skilton ${ }^{5}$, Jane Koleff', Peter Vuillermin ${ }^{1,2,4^{*}+}$, David Burgner ${ }^{1,3+}$ and on behalf of the Barwon Infant Study investigator group

\begin{abstract}
Background: Aortic intima-media thickness measured by transabdominal ultrasound (alMT) is an intermediate phenotype of cardiovascular risk. We aimed to (1) investigate the reproducibility of alMT in a population-derived cohort of infants; (2) establish the distribution of alMT in early infancy; (3) compare measurement by edge-detection software to that by manual sonographic calipers; and (4) assess the effect of individual and environmental variables on image quality.
\end{abstract}

Methods: Participants were term infants recruited to a population-derived birth cohort study. Transabdominal ultrasound was performed at six weeks of age by one of two trained operators. Thirty participants had ultrasounds performed by both operators on the same day. Data were collected on environmental (infant sleeping, presence of a sibling, use of sucrose, timing during study visit) and individual (post-conception age, weight, gender) variables. Two readers assessed image quality and measured alMT by edge-detection software and a subset by manual sonographic calipers. Measurements were repeated by the same reader and between readers to obtain intra-observer and inter-observer reliability.

Results: Aortic IMT was measured successfully using edge-detection in 814 infants, and 290 of these infants also had alMT measured using manual sonographic calipers. The intra-reader intra-class correlation (ICC) $(n=20)$ was 0.90 (95\% Cl 0.76, 0.96), mean difference $1.5 \mu \mathrm{m}$ (95\% LOA -39, 59). The between reader ICC using edge-detection $(n=20)$ was $0.92(95 \% \mathrm{Cl} 0.82,0.97)$ mean difference $2 \mu \mathrm{m}(95 \% \mathrm{LOA}-45.0,49.0)$ and with manual caliper measurement $(n=290)$ the ICC was $0.84(95 \% \mathrm{Cl} 0.80,0.87)$ mean difference $5 \mu \mathrm{m}$ (95\% LOA -51.8, 61.8). Edge-detection measurements were greater than those from manual sonographic calipers (mean alMT $618 \mu \mathrm{m}$ (50) versus mean alMT $563 \mu \mathrm{m}$ (49) respectively; $p<0.001$, mean difference $44 \mu \mathrm{m}, 95 \%$ LOA $-54,142$ ). With the exception of infant crying ( $p=0.001)$, no associations were observed between individual and environmental variables and image quality.

Conclusion: In a population-derived cohort of term infants, alMT measurement has a high level of intra and inter-reader reproducibility. Measurement of aIMT using edge-detection software gives higher inter-reader ICC than manual sonographic calipers. Image quality is not substantially affected by individual and environmental factors.

Keywords: Aortic intima-media thickness, Edge-detection software, Newborn, Atherosclerosis, Fetal origins of disease

\footnotetext{
* Correspondence: peter.vuillermin@deakin.edu.au

${ }^{\dagger}$ Equal contributors

${ }^{1}$ Murdoch Childrens Research Institute, Royal Children's Hospital, Parkville,

Australia

${ }^{2}$ Child Health Research Unit, Barwon Health, Geelong, Australia

Full list of author information is available at the end of the article
}

\section{() Biomed Central}

(c) 2014 McCloskey et al.; licensee BioMed Central Ltd. This is an Open Access article distributed under the terms of the Creative Commons Attribution License (http://creativecommons.org/licenses/by/4.0), which permits unrestricted use, distribution, and reproduction in any medium, provided the original work is properly credited. The Creative Commons Public Domain Dedication waiver (http://creativecommons.org/publicdomain/zero/1.0/) applies to the data made available in this article, unless otherwise stated. 


\section{Introduction}

Atherosclerosis, the pathological basis of cardiovascular disease, has its origins in the neonatal period [1-3]. Research into the fetal origins of adult-onset cardiovascular disease is increasingly using aortic intima-media thickness as measured by trans-abdominal ultrasound (aIMT) as a putative intermediate phenotype of cardiovascular risk [4]. Aortic IMT has shown to be superior to carotid IMT in both feasibility and association with known cardiovascular risk factors $[5,6]$. Small exposure-specific studies in newborn infants have reported associations between increased aIMT and cardiovascular risk factors such as intrauterine growth restriction (IUGR), macrosomia, maternal diabetes, maternal hypercholesterolaemia, and maternal smoking [7-10].

Post mortem studies have shown varying degrees of diffuse intimal thickness present in arteries prone to atherosclerosis from birth $[1,11,12]$. Ultrasound measurement of intima-media has strongly correlated with histological thickness vessels prone to atherosclerosis, in particular carotid and coronary arteries [13,14]. The reproducibility of newborn aIMT as measured by abdominal ultrasound has only been established in small, tertiary hospital-based studies using specialist vascular ultrasonographers $[7,9,10]$. Reported intra-class correlations (ICC) between ultrasound readers are high $(\beta=0.89-0.94)[7,8,15,16]$; the largest reported mean difference between sonographers was $16 \mu \mathrm{m}$ [17]. These results are encouraging, but their generalisability, especially to population-based studies conducted outside the tertiary setting, is uncertain.

To date the only normative data regarding aIMT during early infancy are from two small studies conducted in term infants on days $1-4$ of life. These studies used different inclusion criteria and reported substantial differences in mean values and variation in their aIMT measurements $[15,16]$. Furthermore, these data are different from the distributions presented in other studies of infants at the same age (1-4 days) that compared specific exposure groups (e.g. IUGR infants) to 'normal' infants as controls $[7,9,10]$. Published data only pertain to the first few days of life and the distribution of aortic IMT in older infants has not been reported. Given these limitations, it is relevant to establish the distribution of aIMT in a large, population-derived cohort over the first weeks to months of life. To our knowledge there have been no reported data on the effect of environmental and individual variables on image quality in early infancy or childhood.

This study aimed to establish the reproducibility and distribution of aIMT during early infancy in a birth cohort study conducted in a community setting. In addition it aimed to compare the aIMT measures obtained using edge-detection software and manual sonographic calipers and investigate whether image quality is affected by individual and environmental variables.

\section{Materials and methods}

The Barwon Infant Study (BIS) is a population-derived cohort of 1069 infants recruited prior to 33 weeks gestation. Infants were excluded from BIS if they developed a serious illness in the first few days of life, or if they had major congenital malformations or genetic abnormalities. For the current analysis, preterm infants $(<37$ weeks gestational age) were excluded.

\section{Aortic IMT measurement by abdominal ultrasound}

Infant aIMT was measured at the 6 week study visit (median age 5.9 weeks [5.1-7.0]). One of two research nurses (operators), trained to perform aortic ultrasounds, conducted the imaging using a GE Vivid I ultrasound machine with a 4-13 MHz linear array vascular transducer. The infant was settled in a quiet room with a parent present. A subset of infants also had a sibling present at the ultrasound. Some infants were given sucrose at operator and parental discretion. Aortic images were obtained in accordance with a standard operating procedure $[10,18]$. The ultrasound settings were standardised by using presets and images acquired with simultaneous three-lead ECG gating. The abdominal aorta was first identified in cross-section, just above the umbilicus. A longitudinal, straight, unbranched $1 \mathrm{~cm}$ segment of abdominal aorta proximal to the abdominal bifurcation was captured between the umbilicus and xiphisternum, using a standard protocol $[10,18]$. Following identification of both aortic walls, for the assessment of aIMT, the gain and Time Gain Compensation (TGC) settings were used to optimise the image quality. The images were magnified using a resolution box and three continuous cineloops of five or more cardiac cycles were captured and the images stored digitally for off-line analysis.

\section{Reproducibility, distribution and comparison between measurement methods}

Thirty infants had aortic ultrasounds performed by both ultrasound operators on the same day to allow for measurement of reproducibility between operators.

Using edge-detection software (Carotid Analyzer for Research, version 6, Medical Imaging Applications LLC, Iowa), aIMT was measured offline on all infant ultrasounds by one of two readers (who were not ultrasound operators). The best three to five end-diastolic images per infant (timed on the $\mathrm{R}$ wave) were analysed. The measurements relied on the edge-detection software identifying the aIMT 'edge' along a straight segment of posterior wall, of minimum $4 \mathrm{~mm}$ length (Figure 1 ). The edge-detection software generated a minimum, mean and maximum aIMT for the segment selected. The average of each of these values for an infant's end-diastolic images was taken as the measure for each infant. In order to calculate the intra-reader (test retest) and inter-reader repeatability of 


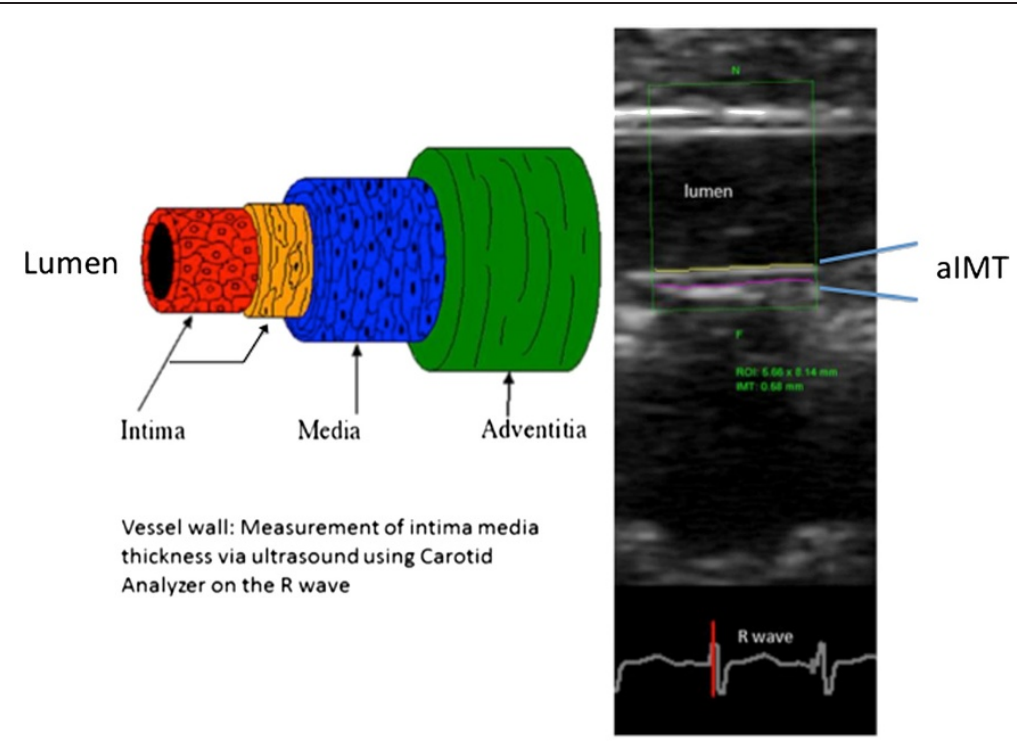

Figure 1 alMT measured using edge-detection software, adapted from [19].

measurements conducted using the edge-detection software, one reader repeated measures in a randomly selected 20 subjects and both readers conducted measures among a separate randomly selected sample of 20 subjects.

In a subset of 290 infants, aIMT was additionally measured using both edge-detection software (as above) and manual sonographic calipers from GE Echopac software. The sonographic caliper measurements were replicated among all 290 infants by two separate readers. Each reader completed 10-20 measures along the aortic wall from consecutive $\mathrm{R}$ waves and from at least two cineloops per child to generate an overall mean aIMT. A difference in aIMT of $>50 \mu \mathrm{m}$ on the first measures between readers prompted the aIMT to be re-measured by both readers. If the difference remained $>50 \mu \mathrm{m}$ on repeat measurement, the repeat measurements were taken as final. The average of both readers' aIMT was taken as the final mean aIMT measurement for each infant. Maximum and minimum aIMT were unable to be measured using this method.

\section{Individual and environmental variables}

The operators recorded the following individual variables; gestation, post-gestational age, age at scan, infant weight, length and head circumference. In addition, environmental variables such as whether the infant was asleep, crying, presence of a sibling, use of sucrose, and timing with other aspects of the overall study protocol were also recorded.

The quality of the aortic cineloops was assessed offline by two readers. Readers were blinded to birth data, individual and environmental variables. Each ultrasound was classified as 'good' (both anterior and posterior wall clearly visible throughout the cardiac cycle), 'adequate' (posterior wall clearly visible throughout the cardiac cycle) or 'poor' (posterior wall intermittently visible, but clearly visible at the time of the $\mathrm{R}$ wave). The subsequent reproducibility analyses included all cineloops regardless of this quality classification.

\section{Statistical methods}

The reproducibility (inter-observer variability) of obtaining aIMT measurements between both operators and readers, as well as the intra-reader (test-retest) variability was assessed using ICC and Bland-Altman plots. Associations between the image quality classification and individual and environmental variables were assessed using chi-square test and one-way ANOVA. Statistical analysis was performed using Stata 12.1 (Stata Corp, College Station, TX).

The Barwon Health Human Research Ethics committee approved the study.

\section{Results}

Of the 1069 eligible infants in BIS, 978 (91\%) completed their six week visit, during which 844 (86\%) had aIMT measured successfully. Excluding preterm infants, aIMT data were available from 814 infants. Five hundred and seventy three of the 814 aIMT images (70\%) were assessed as either 'good' or 'adequate' quality. Gender was evenly represented (53\% male), and the average birth weight was $3.5 \mathrm{~kg}$, approximately the $50^{\text {th }}$ centile in the population [20]. In addition, 290 (35\%) infants had aIMT measured using sonographic calipers; these infants were similar to the main study cohort (Table 1). 
Table 1 Comparison of the total cohort of the study and the sub-cohort who had additional measurements of aIMT performed using manual sonographic calipers

\begin{tabular}{lll}
\hline Variables & $\begin{array}{l}\text { Total Cohort } \\
(\mathbf{n = ~ 8 1 4 )}\end{array}$ & $\begin{array}{l}\text { Sub-cohort* } \\
(\mathbf{n = 2 9 0 )}\end{array}$ \\
\hline Sex (male) & $433(53 \%)$ & $150(51 \%)$ \\
Gestation at birth (weeks) & Mean 39.4 (1.2) & Mean 39.2 (1.2) \\
Age at time of scan (weeks) & Median 5.9 [5.1-7.0] & Mean 5.6 [4.9-6.1] \\
Birth weight (kg) & Mean 3.6 (0.5) & Mean 3.6 (0.5) \\
\hline
\end{tabular}

*Subcohort had alMT measured using both edge-detection and caliper methods.

\section{Reproducibility of measurements between operators and readers}

In the 30 infants who had ultrasounds performed by both operators, the between-operator ICC for the aIMT (measured by a single reader) using edge-detection measurement was 0.84 (95\% CI 0.67, 0.92 mean difference $10 \mu \mathrm{m} 95 \%$ limits of agreement (LOA) -39, 59), and manual caliper measurement 0.87 (95\% CI 0.73, 0.94 mean difference $0.8 \mu \mathrm{m} \mathrm{95 \%} \mathrm{LOA} \mathrm{-50.2,} \mathrm{51.8)} \mathrm{(Figure} \mathrm{2).}$

Aortic IMT was measured offline by one of two readers using edge-detection software. The intra-reader (test retest) ICC ( $n=20)$ was 0.90 (95\% CI 0.76,0.96, mean difference $1.5 \mu \mathrm{m}$ 95\% LOA -45.0, 49). Twenty infants also had aIMT measured by both readers. The inter-reader ICC for edge-detection was 0.92 (95\% CI 0.82, 0.97, mean difference $2 \mu \mathrm{m}$ 95\% LOA -45.0, 49.0). In addition, aIMT was measured in a subcohort of 290 infants by both readers using manual caliper measures. Of these 290 infants who

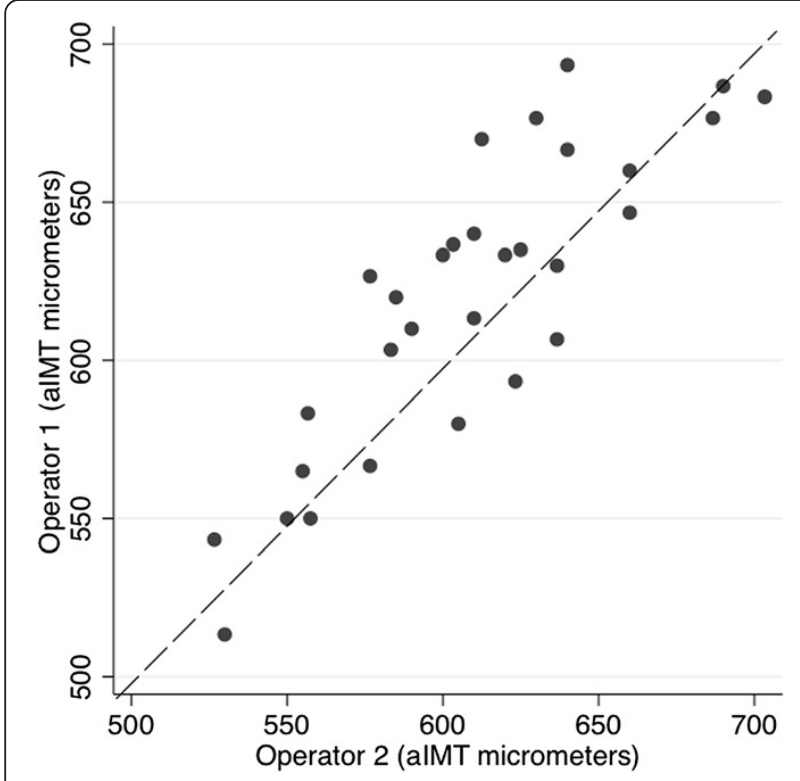

Figure 2 Comparison of aIMT between ultrasounds performed by separate operators on the same day, using edge-detection software, measured by a single reader. ICC $=0.84$, dashed line $y=x$. had their aIMT measured by manual caliper measurement, $42(14 \%)$ had a discrepancy of $>50 \mu \mathrm{m}$ between readers and required repeat measurement. Using the repeat measures as final, the inter-reader ICC for caliper measures was 0.84 (95\% CI 0.80-0.87, mean difference $5 \mu \mathrm{m} 95 \%$ LOA -51.8, 61.8) (Table 2).

Bland Altman analysis of both edge detection versus caliper methods, as well as inter-operator results and inter-reader results using each method, showed no variation between the mean difference and 95\% limits of agreement across the spectrum of results (Figure 3).

\section{Distribution of alMT using edge-detection and sonographic caliper techniques}

Aortic IMT measurements by edge-detection and manual sonographic calipers each visually approximately followed a normal distribution (Figure 4). The aIMT values obtained using edge-detection were higher (edge-detection mean $618 \mu \mathrm{m}$ (50), caliper mean $563 \mu \mathrm{m},(51) \mathrm{p}<0.001$, mean difference $45 \mu \mathrm{m}, 95 \%$ LOA -54, 142) (Figure 4).

Aortic IMT measured by edge-detection increased with age at scan (estimated change per week of age $4.82 \mu \mathrm{m}$, $\mathrm{p}<0.001$ ). Manual sonographic caliper measurement showed a similar association with aIMT, however with borderline significance (change per week of age $4.09 \mu \mathrm{m}$, $\mathrm{p}=0.074)$.

\section{Effect of individual and environmental variables}

Infant individual and environmental variables were collected on 500 infants. Infants who were crying were more likely to have poorer images than those who were not $(38 \%$ versus $23 \%, \mathrm{p}=0.001)$. However there was no evidence of an association between other individual and environmental variables and the quality of the images: sleeping $(\mathrm{p}=0.11)$, presence of sibling $(p=0.35)$, gender $(p=0.45)$, gestational age at birth $(\mathrm{p}=0.84)$, corrected gestational age at scan $(\mathrm{p}=0.24)$, post-natal age at scan $(\mathrm{p}=0.140)$, birth weight $(\mathrm{p}=0.96)$, timing of the scan in relation to lung function testing $(\mathrm{p}=0.34)$, and sucrose use $(\mathrm{p}=0.22)$.

\section{Discussion}

We have demonstrated that aIMT is reproducible in a large community-based study. There was found high intra and inter-operator ICC between (a) the results obtained using paired ultrasound cineloops obtained by two operators and (b) off-line edge-detection measurements obtained from two readers using the same cineloops, using either edge-detection software or manual caliper measurements. In term infants assessed at 6 weeks, there were differences in aIMT values obtained using different measurement methods; mean aIMT was greater using edge-detection compared to manual sonographic calipers. Furthermore, there was no need for repeat measurements using edge-detection software. With the exception of infant 
Table 2 Comparison of the intra-class correlation and mean difference for measurements obtained by edge-detection software and manual caliper measurement

\begin{tabular}{|c|c|c|c|}
\hline & \multicolumn{3}{|c|}{ Measurement method } \\
\hline & Edge detection & & Manual caliper \\
\hline \multicolumn{4}{|c|}{ Inter-operator reliability - same reader using cineloops from different ultrasound operators } \\
\hline \multirow[t]{2}{*}{$n=30$} & ICC $0.84(95 \%$ Cl 0.67, 0.92) & $\mathrm{n}=30$ & ICC $0.87(95 \%$ Cl 0.73, 0.94) \\
\hline & mean diff $10 \mu \mathrm{m}(95 \%$ LOA $-39,59)$ & & mean diff $0.8 \mu \mathrm{m}(95 \% \mathrm{LOA}-50.2,51.8)$ \\
\hline \multicolumn{4}{|c|}{ Inter-reader reliability - two readers using same ultrasound images } \\
\hline \multirow[t]{2}{*}{$n=20$} & ICC $0.92(95 \%$ Cl 0.82, 0.97) & $n=290$ & ICC $0.84(95 \%$ Cl 0.80, 0.87) \\
\hline & mean diff $2 \mu \mathrm{m}(95 \%$ LOA $-45.0,49.0)$ & & mean diff $5 \mu \mathrm{m}(95 \%$ LOA $-51.8,61.8)$ \\
\hline \multicolumn{4}{|c|}{ Intra-reader reliability - same ultrasound images read twice by the same reader } \\
\hline \multirow[t]{2}{*}{$n=20$} & ICC $0.90(95 \%$ Cl 0.76, 0.96) & & N/A (all read by both readers) \\
\hline & mean diff $1.5 \mu \mathrm{m}(95 \% \mathrm{LOA}-45.5,48.5)$ & & \\
\hline
\end{tabular}
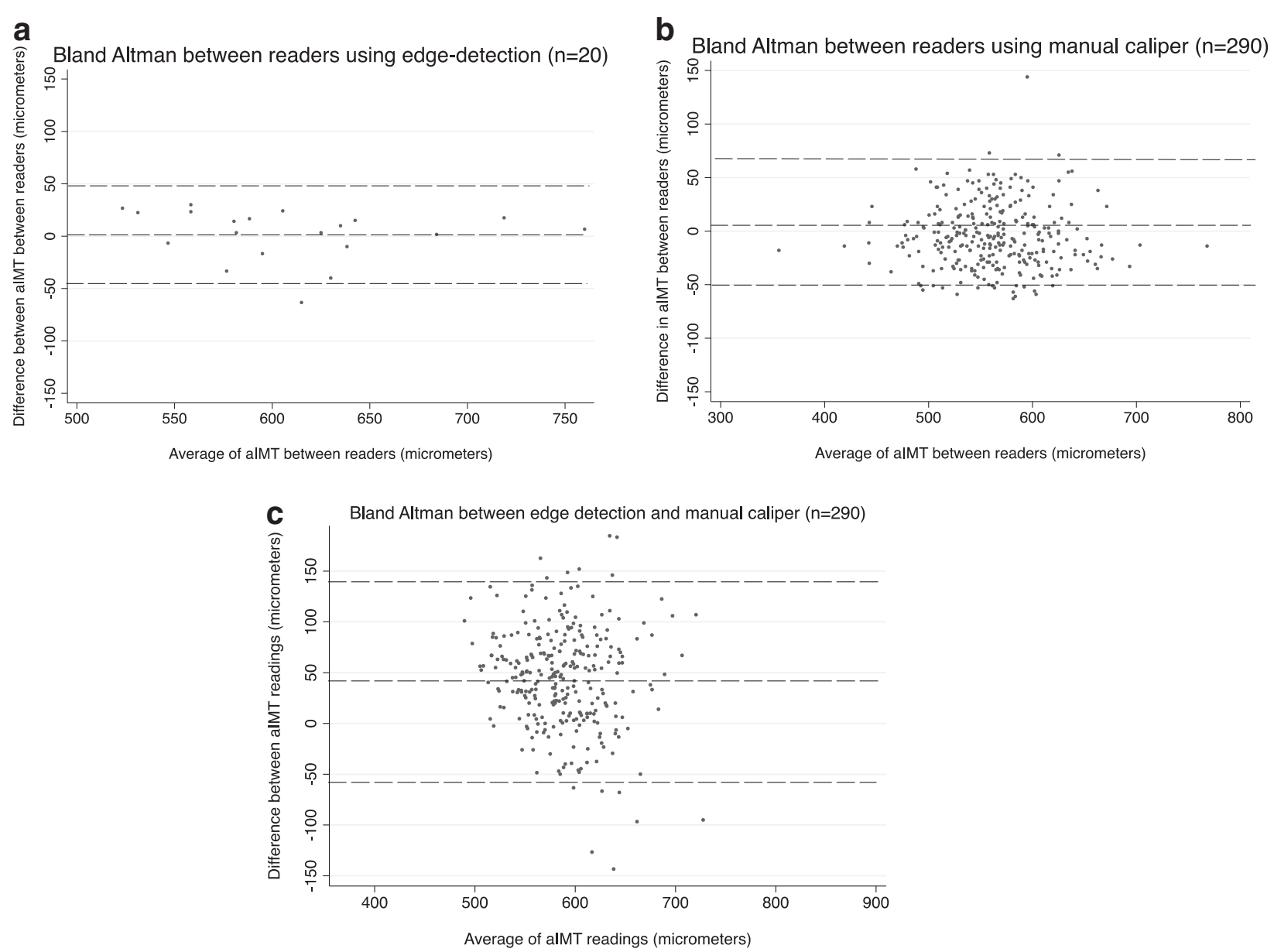

Figure 3 Bland-Altman plots for agreement between readers in aortic IMT measured by (a) edge-detection (b) sonographic calipers and (c) direct comparison of measurements obtained from edge-detection and caliper methods. Dashed lines indicate $95 \%$ limits of agreement. 

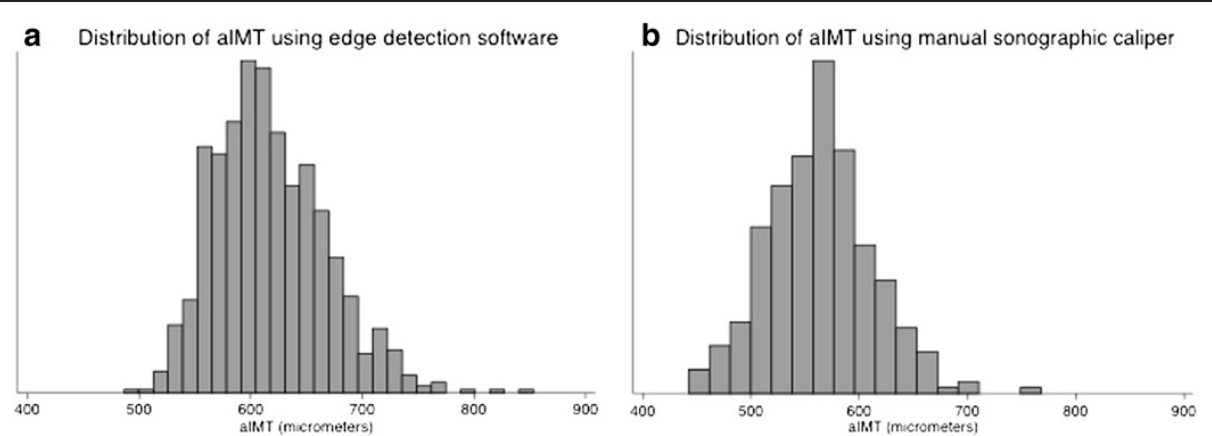

Figure 4 Distribution of alMT with data obtained using (a) edge-detection mean $618 \mu \mathrm{m}(50)$ and (b) manual sonographic calipers mean $563 \mu \mathrm{m}(51)$.

crying, individual and environmental factors did not impact on the quality of images obtained. There was a trend of association between increasing age at scan and higher aIMT measurement.

Previous studies have demonstrated that aIMT is a reproducible measure when conducted by specialist sonographers in a tertiary setting. We have extended these findings by demonstrating that a high reproducibility can be obtained by general research staff following a period of training in the technique. The ICC between research operators performing repeated ultrasounds and readers measuring the same image are consistent with the ICC reported by other groups $[7,9,10,16]$. Indeed the reproducibility of aIMT measures observed is similar to that reported for adult carotid IMT, an integral part of cardiovascular risk assessment [4]. These findings have important feasibility implications for future populationbased research investigating the early life origins of cardiovascular disease.

Our study was able to assess reproducibility but not the dimension of predictive validity -that is we are unable to compare our results to "true" histological measurements of the newborn aorta. We must use intra-observer (test-retest) and inter-observer variability as a proxy for accuracy. A recent study looking at porcine histology as well as aIMT in young children suggested that the use of very-high frequency ultrasound $(25-30 \mathrm{~Hz})$ in infants and young children produced more accurate results than high frequency ultrasound may produce more accurate results than high frequency ultrasound, as used in this study [21]. Introducing another method of assessment with undoubtedly make comparisons between study results even more difficult.

This study provides the first normal data for healthy infant aIMT data for 6 weeks of age. Values described as 'normal' for newborns in the first days of life have been obtained from small studies $(\mathrm{n}<100)$ that have used selected sampling frames and varying definitions of 'normal' that are unlikely to be fully representative of the population. For example, a study by Hondappanavar et al. measured
aIMT in 100 term infants, but excluded all those below the $50^{\text {th }}$ centile for birth weight [16]. Another study by Koklu et al. investigated preterm and term infants of greater than 24 weeks gestational age, including 60 term infants [15]. This study excluded any infants with putative risk factors for increased aIMT (congenital abnormality, IUGR, macrosomia, maternal smoking, abnormal lipid profiles, maternal dyslipidaemia, hypertension or maternal history of cardiovascular disease) [15]. In contrast, our study was more inclusive; only preterm infants and those with congenital abnormalities or significant neonatal illness were excluded.

In addition to sampling considerations, reported newborn values of mean aIMT are highly variable. Variability may arise due to differences in ultrasound equipment, transducer frequency, and, as our study demonstrates, measurement methods. Using manual sonographic calipers, the following studies reported substantial variation. Whilst Koklu et al. $(n=60)$ reported a mean of $385 \mu \mathrm{m}$ with SD 19 at term [15], Hondappanavar et al. $(\mathrm{n}=100)$ found a mean $510 \mu \mathrm{m}$ (41) [16]. Other data are available from healthy control groups in exposure-specific studies that again used sonographic calipers for measurement. For example Koklu et al. reported, in two separate exposurespecific studies, control values of $400 \mu \mathrm{m}(30)(\mathrm{n}=40)$ [9] and $390 \mu \mathrm{m}(30)(\mathrm{n}=30)$ [7]. In contrast Skilton et al. $(n=25)$ [10] used edge-detection software and reported control values of $534 \mu \mathrm{m}$ (58) [10]. Surprisingly, the highest control aIMT measures are from Zanardo's in utero study $(\mathrm{n}=21)$ of infants at 32 weeks gestation which reported mean $1050 \mu \mathrm{m}$ (190) [22]. Zanardo et al. used caliper measurement, but a lower frequency transducer to other studies (3.5-5 Hz versus 8-13 MHz/3-12 MHz). Clearly there are also other significant differences with antenatal imaging such as the depth of imaging, angle of insonation and attenuation that will alter the resolution of images. Our data demonstrate important limitations when comparing results between studies that are not obtained using identical methods. Furthermore, similar to adult studies [23], we show that edge-detection software is at least equivalent to manual caliper measurement 
in reproducibility (higher ICC) and superior in ease of measurement. Ideally, all future studies would adopt the same ultrasound and measurement protocol to allow accurate comparisons between study results.

In contrast to previous studies that examined infants aged 0-4 days, our study participants varied in age around a median of 5.9 weeks [5.1-7.0]. Aortic IMT has previously been reported to increase with both gestation [15] and age [24]. Concordantly, we found a positive association between aIMT measured by edge-detection software and age at time of scan. Thus the potential influence of gestation and age should be considered when investigating the determinants of aIMT during early life and analyses should be adjusted accordingly.

With the exception of infant crying, we found no evidence that individual and environmental variables, including timing of ultrasound scans during study visits, affected image quality. The effect of environmental variables on image quality is particularly relevant beyond the first days of life, as infants become progressively increasingly more physically active, potentially affecting the quality of the ultrasound images. Our measures of aIMT occurred in the context of multiple other research procedures, such as anthropometric and lung function measurements, and this is likely to the case for many studies of a similar nature to BIS. These results reinforce the feasibility of newborn aIMT measurement in a community-based study where other measures are being taken during a single visit.

\section{Conclusion}

Aortic IMT is a highly reproducible measure that is suitable for use in population-based studies of cardiovascular health and development. In 6 week old infants, aIMT approximates a normal distribution. Results differ depending on the measurement technique (edge-detection vs manual sonographic calipers), so it is imperative that a uniform technique is employed throughout. Edge-detection software has superior reproducibility to manual sonographic caliper measurements. With the exception of infant crying, there is no evidence that image quality is affected by infant behavioural and environmental variables.

\section{Abbreviations \\ IMT: Intima-media thickness; alMT: Aortic intima-media thickness; CIMT: Carotid intima-media thickness; ICC: Intra-class correlation; 95\% LOA: $95 \%$ limits of agreement; $95 \% \mathrm{Cl}$ : 95\% confidence interval.}

\section{Competing interests}

The authors declare that they have no competing interests.

\section{Authors' contributions}

KM was one of the ultrasound and first author. A-L P was involved in study design and drafting. JC was involved in statistical support and drafting. KJ was involved in statistical support and drafting. MC was involved in study design and drafting. MS was involved in the study protocol, design and drafting. JK was one of the readers and involved teaching ultrasound technique. PV was involved in the overall BIS design, study development, funding and drafting. DB was involved in study design, funding and drafting. All authors read and approved the final manuscript.

\section{Acknowledgments}

We would like to acknowledge and thank the BIS research staff and participants.

\section{Barwon Infant Study investigator group}

Peter Vuillermin: Barwon Health, Geelong, Australia; The Murdoch Childrens Research Institute, Parkville, Austrlalia; Deakin University, Geelong, Australia; The University of Melbourne, Parkville, Australia.

Anne-Louise Ponsonby: The Murdoch Childrens Research Institute, Parkville, Austrlalia; The University of Melbourne, Parkville, Australia.

John Carlin: The Murdoch Childrens Research Institute, Parkville, Austrlalia; The University of Melbourne, Parkville, Australia.

Katie Allen: The Murdoch Childrens Research Institute, Parkville, Austrlalia; The University of Melbourne, Parkville, Australia; The Royal Children's Hospital, Parkville, Australia.

Mimi Tang: The Murdoch Childrens Research Institute, Parkville, Austrlalia; The University of Melbourne, Parkville, Australia; The Royal Children's Hospital, Parkville, Australia.

Richard Saffery: The Murdoch Childrens Research Institute, Parkville, Austrlalia; The University of Melbourne, Parkville, Australia.

Sarath Ranganathan: The Murdoch Childrens Research Institute, Parkville, Austrlalia; The University of Melbourne, Parkville, Australia; The Royal Children's Hospital, Parkville, Australia.

David Burgner: The Murdoch Childrens Research Institute, Parkville, Austrlalia; The University of Melbourne, Parkville, Australia.

Terry Dwyer

Kim Jachno: The Murdoch Childrens Research Institute, Parkville, Austrlalia. Peter Sly

\section{Funding source}

The Barwon Infant Study is supported by a National Health and Medical Research Council (NHMRC) Project Grant and the Victorian Government's Operational Infrastructure Support Program.

KM is supported by Sidney Myer PhD scholarship.

ALP and DPB are supported by a NHMRC Senior Research Fellowship and

Honorary National Heart Foundation Future Leader Fellowship.

The Heart Research Group at the MCRI is supported by Big W and RCH 1000, $\mathrm{RCH}$ Foundation.

\section{Author details}

'Murdoch Childrens Research Institute, Royal Children's Hospital, Parkville, Australia. ${ }^{2}$ Child Health Research Unit, Barwon Health, Geelong, Australia. ${ }^{3}$ University of Melbourne, Parkville, Australia. ${ }^{4}$ Deakin University, Burwood, Australia. ${ }^{5}$ Boden Institute of Obesity, Nutrition, Exercise and Eating Disorders, University of Sydney, Sydney, Australia.

Received: 13 April 2014 Accepted: 9 May 2014

Published: 3 June 2014

\section{References}

1. Stary HC: Lipid and macrophage accumulations in arteries of children and the development of atherosclerosis. Am J Clin Nutr 2000, 72(5 Suppl):1297S-1306S.

2. McGill HC Jr, McMahan CA, Herderick EE, Tracy RE, Malcom GT, Zieske AW, Strong JP: Effects of coronary heart disease risk factors on atherosclerosis of selected regions of the aorta and right coronary artery. PDAY research group. Pathobiological determinants of atherosclerosis in youth. Arterioscler Thromb Vasc Biol 2000, 20(3):836-845.

3. Napoli C, D'Armiento FP, Mancini FP, Postiglione A, Witztum JL, Palumbo G, Palinski W: Fatty streak formation occurs in human fetal aortas and is greatly enhanced by maternal hypercholesterolemia. Intimal accumulation of low density lipoprotein and its oxidation precede monocyte recruitment into early atherosclerotic lesions. J Clin Invest 1997, 100(11):2680-2690.

4. Urbina EM, Williams RV, Alpert BS, Collins RT, Daniels SR, Hayman L, Jacobson M, Mahoney L, Mietus-Snyder M, Rocchini A, Steinberger J, McCrindle B: Noninvasive assessment of subclinical atherosclerosis in children and adolescents: recommendations for standard assessment for 
clinical research: a scientific statement from the American heart association. Hypertension 2009, 54(5):919-950.

5. Dawson JD, Sonka M, Blecha MB, Lin W, Davis PH: Risk factors associated with aortic and carotid intima-media thickness in adolescents and young adults: the Muscatine offspring study. J Am Coll Cardiol 2009, 53(24):2273-2279.

6. McCloskey K, Vuillermin P, Ponsonby AL, Cheung M, Skilton MR, Burgner D: Aortic intima-media thickness measured by trans-abdominal ultrasound as an early life marker of subclinical atherosclerosis. Acta Paediatr 2014, 103(2):124-130. Epub 2013 Dec 16.

7. Koklu E, Akcakus M, Kurtoglu S, Koklu S, Yikilmaz A, Coskun A, Gunes T: Aortic intima-media thickness and lipid profile in macrosomic newborns. Eur J Pediatr 2007, 166(4):333-338.

8. Gunes T, Koklu E, Yikilmaz A, Ozturk MA, Akcakus M, Kurtoglu S, Coskun A, Koklu S: Influence of maternal smoking on neonatal aortic intima-media thickness, serum IGF-I and IGFBP-3 levels. Eur J Pediatr 2007, 166(10):1039-1044

9. Koklu E, Kurtoglu S, Akcakus M, Koklu S, Buyukkayhan D, Gumus H, Yikilmaz A: Increased aortic intima-media thickness is related to lipid profile in newborns with intrauterine growth restriction. Horm Res 2006 65(6):269-275.

10. Skilton MR, Evans N, Griffiths KA, Harmer JA, Celermajer DS: Aortic wall thickness in newborns with intrauterine growth restriction. Lancet 2005, 365(9469):1484-1486.

11. Lo Vasco VR, Salmaso R, Zanardo V, Businaro R, Visentin S, Trevisanuto D, Cosmi E: Fetal aorta wall inflammation in ultrasound-detected aortic intima/media thickness and growth retardation. J Reprod Immunol 2011, 91(1-2):103-107.

12. Nakashima $Y$, Chen $Y X$, Kinukawa N, Sueishi K: Distributions of diffuse intimal thickening in human arteries: preferential expression in atherosclerosis-prone arteries from an early age. Virchows Arch 2002, 441(3):279-288.

13. Wong M, Edelstein J, Wollman J, Bond MG: Ultrasonic-pathological comparison of the human arterial wall. Verification of intima-media thickness. Arteriosd Thromb J Vasc Biol Am Heart Assoc 1993, 13(4):482-486.

14. Farkas SMS, Nagy K, Hortobagyi T, Csiba L: Comparative in vivo and in vitro postmortem ultrasound assessment of intima-media thickness with additional histological analysis in human carotid arteries. Perspect Med 2012, 1(1-12):170-176.

15. Koklu E, Kurtoglu S, Akcakus M, Yikilmaz A, Coskun A, Gunes T: Intima-media thickness of the abdominal aorta of neonate with different gestational ages. J Clin Ultrasound 2007, 35(9):491-497.

16. Hondappanavar A, Sodhi KS, Dutta S, Saxena AK, Khandelwal N: Quantitative ultrasound measurement of intima-media thickness of abdominal aorta and common carotid arteries in normal term newborns. Pediatr Cardiol 2013, 34(2):364-369. Epub 2012 Aug 15.

17. Akcakus M, Koklu E, Baykan A, Yikilmaz A, Coskun A, Gunes T, Kurtoglu S, Narin N: Macrosomic newborns of diabetic mothers are associated with increased aortic intima-media thickness and lipid concentrations. Horm Res 2007, 67(6):277-283.

18. Jarvisalo MJ, Jartti L, Nanto-Salonen K, Irjala K, Ronnemaa T, Hartiala Jر, Celermajer DS, Raitakari OT: Increased aortic intima-media thickness: a marker of preclinical atherosclerosis in high-risk children. Circulation 2001, 104(24):2943-2947.

19. West JL: Biomimetic Strategies in Vascular Tissue Engineering. In Frontiers of Engineering: Reports on Leading-Edge Engineering from the 2004 NAE Symposium on Frontiers of Engineering. Washington, DC: The National Academies Press; 2005:56.

20. Dobbins TA, Sullivan EA, Roberts $C L$, Simpson JM: Australian national birthweight percentiles by sex and gestational age, 1998-2007. Med J Aust 2012, 197(5):291-294.

21. Sarkola T, Slorach C, Hui W, Bradley TJ, Redington AN, Jaeggi E: Transcutaneous very-high resolution ultrasound for the quantification of carotid arterial intima-media thickness in children - feasibility and comparison with conventional high resolution vascular ultrasound imaging. Atherosclerosis 2012, 224(1):102-107.

22. Zanardo V, Visentin S, Trevisanuto D, Bertin M, Cavallin F, Cosmi E: Fetal aortic wall thickness: a marker of hypertension in IUGR children? Hypert res Official J Japanese Soc Hypert 2013, 36(5):440-443.
23. Johnny Sundholm TG, Taisto S: Semi-automatic border detection software for the quantification of arterial lumen, intima-media and adventitia layer thickness with very-high resolution ultrasound. Atherosclerosis 2014, 234(2):283-287 [Epub ahead of print].

24. Davis PH, Dawson JD, Blecha MB, Mastbergen RK, Sonka M: Measurement of aortic intimal-medial thickness in adolescents and young adults. Ultrasound Med Biol 2010, 36(4):560-565.

doi:10.1186/1476-7120-12-18

Cite this article as: McCloskey et al.: Reproducibility of aortic intimamedia thickness in infants using edge-detection software and manual caliper measurements. Cardiovascular Ultrasound 2014 12:18.

\section{Submit your next manuscript to BioMed Central and take full advantage of:}

- Convenient online submission

- Thorough peer review

- No space constraints or color figure charges

- Immediate publication on acceptance

- Inclusion in PubMed, CAS, Scopus and Google Scholar

- Research which is freely available for redistribution 